\title{
Ilioinguinal and Iliohypogastric Nerve Block with Anatomical Landmark and Direct Visualization Technique
}

\author{
ZAHRA RAOOFI ${ }^{1}$, MINOODOKHT BAVARSAD KARIMI ${ }^{2}$, SEYEDEH PEGAH PARVAR ${ }^{3}$. \\ SEYEDEH PANIZ PARVAR ${ }^{4}$
}

\begin{abstract}
:
Objective(s): The aim of the study was to evaluate the efficacy of ilioinguinal and iliohypogastric nerve block with anatomical landmark and direct visualization technique on postoperative pain relief and use of opioids after lower segment caesarean section.

Materials and Methods: In a double blinded randomized clinical trial that conducted in Shahid Akbarabadi hospital of Tehran, 150 pregnant women candidate for elective cesarean section included in the study and randomly allocated into two groups (nerve block with bupivacaine $0.25 \%$ and control). Procedure of cesarean section was equal in all cases and patients were assigned randomly to receive nerve block with either $20 \mathrm{ml}$ of $0.25 \%$ bupivacaine (group A-case group) or $20 \mathrm{ml}$ of normal saline solution (group B - placebo group) 2 hours after operation. Opioids dose was measured during the postoperative period during 8 hours. Postoperative pain was assessed at 2, 4, 6, 8 hours postoperatively with a visual analog scale (VAS). Data analyzed by SPSS.
\end{abstract}

Results: There was a significant decrease in opioid administration and pain on visual analog scale in group $A$ (nerve block), as compared with Group (placebo). 8 hours after the operation, pain severity in both case and control groups were similar. Total dose of opioid administration was significantly lower in group $A(p=0.00)$.

Conclusion: This study shows that ilioinguinal and iliohypogastric nerve block with anatomical landmark and direct visualization technique is good substitution for opioid in postoperative pain control in cesarean section

Key words: Bupivacaine, ilioinguinal and iliohypogastric nerve block, cesarean section pain relief.

Introduction:

Postoperative pain may cause many malfunctions in various systems, including increase of sympathetic tone, catecholamine release and increase of catabolic hormones. These alterations may result in sodium and water retention, hypertension and hyperglycemia ${ }^{1}$. Hypercoagulopathy, increase of myocardial oxygen consumption and probably myocardial ischemia, respiratory failure and bowel ileus are other examples of effects of pain on body systems ${ }^{2}$.

Therefore, postoperative pain management is one of main goals in any kind of surgery. There are many routes and methods of postop pain control; like intravenous injection of drugs, local anesthesia and epidural injection of local anesthetics ${ }^{3}$.Opioids are the most famous pain killers in postop pain control. Indeed, because of side effects of opioids like itching, nausea and vomiting and respiratory depression many studies designed to find a good substitute for opioids.

In obstetric and gynecologic surgeries, ilioinguinal and iliohypogastric nerve block with bupivacaine is a good choice for postop pain control; it can cause pain relief for at least 5-7 hours via nerve block ${ }^{4}$.

Previous studies showed that bupivacaine nerve block can cause pain relief in many lower abdominal

1. Assistant professor of Obstetrics and Gynecology, Iran University of Medical Sciences

2. Iran University of Medical Sciences

3. Medical Student. Azad university of Medical Sciences.

4. Medical Student. Kerman University of Medical Sciences. 
surgeries through ultrasonographic localization or blind localization through anatomical landmark ${ }^{5-6}$. The aim of this study is evaluation of the efficacy of ilioinguinal and iliohypogastric nerve block through anatomical landmark and direct visualization technique.

\section{Materials and methods:}

In a double blinded randomized clinical trial 150 women who were candidate for cesarean section in Shaheed Akbarabadi Hospital of Tehran enrolled in study considering inclusion and exclusion criteria. The research protocol was approved by Iran University ethicscommittee.

All steps of study explained for each case and an informed consent was obtained. Exclusion criterias were medical problems like diabetes mellitus, hypertension, hypersensitivity to bupivacaine, coagulopathy or any drug addiction.

All cases randomly allocated into case or control group. Patients and surgeon were not aware of this allocation. All cases underwent cesarean section with spinal anesthesia, which reduced the effect of procedure duration on pain control need. Before abdominal wall closure, two angiocatheter inserted and fixed bilaterally at anatomical landmark point in abdominal wall just above of peritoneal area 2 centimeter medial and 2 centimeter above of anterior superior iliac spine under direct visualization. After the procedure, surgery accomplished well and patient transferred to the obstetric ward.

If patient was allocated in case group,2hours after surgery 20cc of $0.25 \%$ bupivacaine administered through catheters for blocking of nerves and if patient allocated in control group, 20cc of normal saline administered and then angiocatheters were removed. All patients and examiner were blind to the drug administered and syringes were same between two groups.

Pain score of each patient recorded via a visual analog score system at the time of nerve block (2 hours), 4,6 and 8 hours postop.

If patients pain score was equal or over $3,30 \mathrm{mg}$ of pentazoscine administered intramuscularly. If pain control was poor, $30 \mathrm{mg}$ of pentazoscine administered every 1 hour.

Pain score of 2,4,6 and 8 hours postop, amount of pentazoscine administered for each patient, adverse effects of pentazoscine, itching, nausea, vomiting or any complication were recorded in two groups

Data analyzed with SPSS. P value under 0.05 assumed significant.

\section{Results:}

Demographic characteristics in two groups were not significantly different(table1).Pain score based on VAS was between 1 and 4 in case group 2 hours postoperatively and pain score of 1 was most frequent(53.3\%), but in control group this measure was between 1 and 4 and pain score of 2 was the most significant(53.3\%). There is not significant difference between groups $(\mathrm{P}=0.11)$

Pain score of 4 hours postoperative was between 1 and 4 in case group and pain score of 2 was the most frequent(29.3\%) and this measure in control group was between 1 and 5 that the most frequent pain score was 2(49.3\%), the difference between 2 groups was significant $(\mathrm{P}=0.0002)$.

$6^{\text {th }}$ hour postoperative pain score in case group was between 0 and 2 and pain score of zero was the most frequent in case group and in control group pain score of $6^{\text {th }}$ hour postoperative was between 1 to 3 and pain score of 1 was the most frequent (87.7\%) and difference was significant $(\mathrm{P}=0.001)$

$8^{\text {th }}$ hour postoperative pain score in case group was between 0 and 1 and frequent pain score in this group was zero (77.3\%) and in control group was between 0 and 1 and the most frequent score was $68 \%$ that this difference was not significant $(P=0.202)$ (chart 2).

Sixty eight patients (90.66\%) never got pentazoscine in case group and in control group this measure was 23 patients(30.6\%) that difference between 2 groups was significant $(P=0.0001)$.

Frequency of itching in case group was 71 cases $(94.6 \%)$ and in control group was 69 cases(92\%) and difference was not significant $(\mathrm{P}=0.074)$.

Frequency of nausea and vomiting in case group was 5 cases $(6.7 \%)$ and in control group this measure was 12 cases(16\%), difference was not significant $(\mathrm{P}=0.077)$.

There was a case of haematoma of catheter site in control group that resolved with close observation. Signs of local anesthetic toxicity was not observed in any of the cases. 
Table-I

Comparison of demographic and clinical data of cases between 2 groups

\begin{tabular}{lccc}
\hline & $\begin{array}{c}\text { Nerve block group }(\mathrm{n}=72) \\
\text { Mean } \pm \text { SD }\end{array}$ & $\begin{array}{c}\text { Control group }(\mathrm{n}=78) \\
\text { Mean } \pm \text { SD }\end{array}$ & Significance \\
\hline Maternal age(Yrs) & $28.8 \pm 4.6$ & $28.9 \pm 5.3$ & 0.948 \\
Gestational age(Weeks) & $37.2 \pm 2.6$ & $36.8 \pm 2.91$ & 0.825 \\
BMI(kg/m2) & $25.3 \pm 3.5$ & $25.6 \pm 3.9$ & 0.648 \\
GravidityRange (mode) & $1-6(\max 2)$ & $1-5(\max 2)$ & 0.384 \\
ParityRange (mode) & $0-3(\max 1)$ & $0-4(\max 1)$ & 0.185 \\
\hline
\end{tabular}

Table-II

Comparison of pain score between groups

\begin{tabular}{lccc}
\hline Pain score & $\begin{array}{c}\text { Nerve block group }(\mathrm{n}=72) \\
\text { Mean } \pm \text { SD }\end{array}$ & $\begin{array}{c}\text { Control group }(\mathrm{n}=78) \\
\text { Mean } \pm S D\end{array}$ & Significance \\
\hline In 2 hrs. & $1.6 \pm 0.88$ & $1.90 \pm 0.73$ & 0.11 \\
In 4 hrs. & $1.18 \pm 0.92$ & $1.86 \pm 0.89$ & 0.00 \\
In 6 hrs. & $0.52 \pm 0.57$ & $1.2 \pm 0.45$ & 0.00 \\
In 8 hrs. & $0.22 \pm 0.42$ & $0.32 \pm 0.46$ & 0.202 \\
\hline
\end{tabular}

\section{Discussion:}

This study shows that ilioinguinal and iliohypogastric nerve block with anatomical landmark and direct visualization technique is good substitution for opioid in postoperative pain control in cesarean section and may reduce amount of opioid administration postoperatively.

One of conventional postoperative pain control is administration of long acting opioids; they have many side effects like respiratory depression, nausea, vomiting, sedation and itching that reduce their use.

There are many routes and methods of local anesthesia for postoperative pain control; like ultrasonographic guided peripheral nerve blocks, blocks through landmark-based technique and the transversus abdominis plane (TAP) blocks. Castello et al showed that the transversus abdominis plane (TAP) block was not effective in postoperative pain control of cesarean section ${ }^{7}$ but Study of Belavy et al in Australia showed that the US-guided TAP block reduces morphine requirements after caesarean delivery when used as a component of a multimodal analgesic regimen.Dose of morphine was $3.5 \mathrm{mg}$ in nerve block group and 18mg in control group of caesarean section(difference was significant) ${ }^{8}$. Also McDonnell showed that the TAP block, as a component of a multimodal analgesic regimen, provided superior analgesia when compared with placebo block up to 48 post-operative hours after elective caesarean delivery ${ }^{9}$.

Weintraudet al studied ultrasonographic observation of peripheral nerve blocks to determine the actual location of local anesthetic when ilioinguinal/ iliohypogastric nerve blocks are performed using landmark-based techniques in children. Their results showed that in $14 \%$ of the blocks, the local anesthetic was administered correctly around the nerves resulting in successful blocks. In the remaining $86 \%$, the local anesthetic was administered in adjacent anatomical structures (iliac muscle 18\%, transverse abdominal muscle $26 \%$, internal oblique abdominal muscle $29 \%$, external oblique abdominal muscle $9 \%$, subcutaneous $2 \%$, and peritoneum $2 \%$ ), and $45 \%$ of these blocks failed. ${ }^{10}$ Also Demirci A et al showed US guided iliohypogastric/ilioinguinal nerve block in adult inguinal herniorrhaphies provides a more effective analgesia and higher satisfaction of analgesia than iliohypogastric/ilioinguinal nerve block with the anatomical landmark technique. Moreover, it may be suggested that the observation of anatomical structures with the US may increase the success of the block, and minimize the block-related complications ${ }^{6}$. Amory et al reported a case of 
accidental puncture of the small bowel during an ilioinguinal/iliohypogastric nerve block procedure for hernia repair. The diagnosis was made a few days later during a laparoscopic exploration owing to the progressive onset of clinical and radiological intestinal obstruction. A large, obstructing subserosalhaematoma was found without any apparent perforation of the mucosa, and the damaged loop was resected. ${ }^{11}$

The insertion of catheter is very important to get the best result and also less complication. We introduced a new technique for ilioinguinal and iliohypogastric nerve block through combination of anatomical landmark technique and direct visualization before abdominal closure. It can be done during operation with advantages such as palpitation of anatomical landmark when the patient hasn't pain and also the tip of catheter is visible just above the peritoneum. It can reduce the complications such as bowel injury and also is a less expensive one. In the present study postoperative pain score in case group were significantly lower in $4^{\text {th }}$ and $6^{\text {th }}$ hour postoperatively but there is not significant difference in $8^{\text {th }}$ hour postoperative pain score between groups. One limitation of our study was single infiltration and so its effects was finished in 8 hours. In future studies it can be done by multiple infiltration through the same angio catheter and removing it depends on the patient need.

\section{Aknowledgement:}

I would like to thank vice-chancellor for research of Iran university of medical sciences for financial support.

\section{References:}

1. Liu S, Carpenter RL, Neal JM.Epidural anesthesia and analgesia. Their role in postoperative outcome, Anesthesiology 1995; 82:1474.

2. Wu CL, Fleisher LA: Outcomes research in regional anesthesia and analgesia. AnesthAnalg 2000; 91:1232.

3. RobertW. Hurley, Acute Postoperative Pain; Christopher L. Wu: in Ronald D miller Anesthesia: 7th Ed, Philadelphia. Churchill Livingstone, chapter 87, 2010, page 2757-2780.
4. Sweetman SC, ed. Martindale: The complete Drug Reference. 33rd ed. London: Pharmaceutical Press; 2002:135-144.

5. Gligorgucev,Jeffrey lee, bilateral ultrasound guided continuous ilioinguinal/iliohypogastric block for pain relief after cesarean delivery: obstetrics anesth: 2008:106.4.1220-22.

6. Demirci A, Efe EM2, Türker G, et al.Iliohypogastric/ilioinguinal nerve block in inguinal hernia repair for postoperative pain management: comparison of the anatomical landmark and ultrasound guided techniques.Braz J Anesthesiol. 2014 Sep-Oct;64(5):350-6.

7. Costello, Joseph F. MB, FCARCSI; Moore, Albert R. MD, FRCPC; Wieczorek, Paul M. MD, FRCPC et al. The TransversusAbdominis Plane Block, When Used as Part of a Multimodal Regimen Inclusive of Intrathecal Morphine, Does Not Improve Analgesia After Cesarean Delivery.RegAnesth Pain Med. 2009 Nov-Dec; 34(6):586-9.

8. D.Belavy, P.J.Colishaw, ultrasound guide transversus abdominals plane block for block analgesia after cesarean delivery: British Jour Anesth:2009:103:5,726-730.

9. John G McDonnell, Gerard Curley, John Carney et al.The analgesic efficacy of transversus abdominis plane block after cesarean delivery: a randomized controlled trial. Anesth Analg 2008 Jan;106(1):186-91.

10. Weintraud, Marion MD*; Marhofer, Peter MD*; Bösenberg, Adrian MBChB, et al .llioinguinal/ Iliohypogastric Blocks in Children: Where Do We Administer the Local Anesthetic Without Direct Visualization. Anesthesia \& Analgesia 2008; 106: 89-93

11. Amory C, Mariscal A, Guyot E, Chauvet P, Leon A and Poli-Merol ML. Is ilioinguinal/iliohypogastric nerve block always totally safe in children? Pediatric Anesthesia 2003;13: 164-166. 